\title{
Envelope analysis of the human alpha rhythm
}

2 Víctor Manuel Hidalgo ${ }^{1, *}$, Javier Díaz ${ }^{2}$, Jorge Mpodozis ${ }^{1}$, and Juan-Carlos

${ }^{1}$ Biology of Cognition Laboratory, University of Chile

${ }^{2}$ International Institute for Sleep Medicine, University of Tsukuba

*Corresponding author: Víctor Manuel Hidalgo, victor.hidalgo@ug.uchile.cl 


\begin{abstract}
The origin of the human alpha rhythm has been a matter of debate since Lord Adrian attributed it to synchronous neural populations in the occipital cortex. While some authors have pointed out the Gaussian characteristics of the alpha rhythm, their results have been repeatedly disregarded in favor of Adrian's interpretation; even though the first EEG Gaussianity reports can be traced back to the origins of EEG. Here we revisit this problem using the envelope analysis - a method that relies on the fact that the coefficient of variation of the envelope (CVE) for continuous-time zero-mean Gaussian white noise (as well as for any filtered sub-band) is equal to $\sqrt{(4-\pi) / \pi} \approx 0.523$, thus making the CVE a fingerprint for Gaussianity. As a consequence, any significant deviation from Gaussianity is linked to synchronous 16 neural dynamics. Low-CVE signals come from phase-locking dynamics, while mid-CVE signals constitute Gaussian noise. High-CVE signals have been linked to unsteady dynamics in populations of nonlinear oscillators. We analyzed occipital EEG and iEEG data from massive public databases and the order parameter of a population of weakly coupled oscillators using the envelope analysis. Our results showed that the human alpha rhythm can be characterized as a rhythmic, Gaussian, or pulsating signal due to intra- and inter-subject variability. Furthermore, Fourier analysis showed that the canonical spectral peak at $\approx 10[\mathrm{~Hz}]$ is present in all three CVE classes, thus demonstrating that this same peak can be produced by rhythms, Gaussian noise, and pulsating ripples. Alpha-like signals obtained from a population of nonlinear oscillators showed a different CVE depending only on the coupling constant, suggesting that the same neural population can produce the amplitude modulation patterns observed in experimental data. iEEG data, however, was found to be mostly Gaussian, specially the signals recorded from the calcarine cortex. These results suggest that a new interpretation for EEG event-related synchronization/desynchronization (ERS/ERD) may be needed. Envelope analysis constitutes a novel complement to traditional Fourier-based methods for neural signal analysis relating amplitude modulations (CVE) to signal energy.
\end{abstract}

Keywords: EEG, iEEG, alpha rhythm, envelope, neural synchronization, Gaussianity, ERS/ERD, Kuramoto model. 


\section{Introduction}

The neuronal dynamics responsible for the generation of the alpha rhythm has been a matter of attention since its discovery by Berger (1929). Lord Adrian undertook a thorough examination of the alpha rhyhthm and attributed its origin to neural synchronization (Adrian and B. $\mathrm{H}$. Matthews, 1934); an idea that went mainstream rapidly and became the standard interpretation for EEG phenomena. Indeed, neural synchronization is a fundamental mechanism in EEG signal generation (Buzsáki, Anastassiou, and Koch, 2012) and a widespread phenomenon at many levels of the biological organization (Singer, 1999; Varela et al., 2001). Adrian also reported that the rhythm can be entrained by a flicker to frequencies as high as $25[\mathrm{~Hz}]$. In this line, the famous cybernetician Norbert Wiener hypothesized that the alpha rhythm is produced by a synchronous population of coupled nonlinear oscillators with similar natural frequencies (Wiener, 1948; Strogatz, 1994); a claim supported by Dr. Yoshiki Kuramoto's early results (Kuramoto, 1975).

Nevertheless, some authors have reported that the alpha rhythm exhibits Gaussian characteristics - a possibility that has been constantly disregarded for any EEG phenomena under the assumption that the amplitude of a signal emerging from an asynchronous population is either zero or, at best, too low to be measured (Cohen, 2017; Lachaux et al., 1999; Singer, 1999). On the contrary, the amplitude of a signal emerging from a population of asynchronous oscillators is proportional to $\sqrt{n}$ (Elul, 1972), as it was recognized early in the EEG literature by Motokawa (1943). In fact, Dr. Yoiki Motokawa himself contended that the alpha rhythm originated from a population of uncorrelated oscillators (Motokawa and Mita, 1942). In an elegant approach, Saunders (1963) compared the distribution of raw signal amplitude values of "well developed" alpha rhythm epochs against the Gaussian distribution and found a close fit. In this line, some researchers suggested that the underlying neural generator may be better described as an alpha filter (Lopes Da Silva, Van Lierop, et al., 1973): a system that receives random input and produce the characteristic narrow band signal centered $\approx 10[\mathrm{~Hz}]$. This interpretation of the alpha rhythm generator as a bandpass filter can be traced back to Prast (1949). Dick and Vaughn (1970) went one step further and estimated the envelope function by the complex demodulation method. They found a close similarity between the distribution 
of the envelope values of alpha rhythm epochs and the Rayleigh distribution. The envelope function of a Gaussian signal follows the Rayleigh distribution (Schwartz, Bennett, and Stein, 1995). Furthermore, they proposed to study the changes of the variance of the envelope to explore the time-variant behaviour of the alpha rhythm; an approach similar to ours exposed here.

Another remarkable characteristic of the alpha rhythm is what Adrian and Matthews described as "waxing and waning" or "pulsating" activity - i.e. a distinctive amplitude modulation pattern. While sometimes ignored, there is some interindividual variability among alpha amplitude modulation patterns (Niedermeyer, 2005). H. Davis and P. A. Davis (1936) reported as many as four distinct alpha rhythm types based on their amplitude modulation, including cases where little or no waning is present. These different pattern are also reflected in the frequency of the alpha rhythm envelope (Schroeder and Barr, 2000). Consequently, these different alpha modulation regimes result in characteristic raw signal and envelope waveforms. Interestingly, in recent years, some authors have pointed out the importance of the signal waveform to detect features in neural signals that may not be evident using Fourier-based methods (Cole and Voytek, 2017). As we shall expose, the signal waveform does offer information about neural dynamics; information that can be extracted analyzing the envelope signal.

In a previous report, Díaz, Bassi, et al. (2018) used rat EEG to describe a novel framework to characterize EEG signals: the envelope analysis. This analysis relies on the fact that the coefficient of variation of the envelope(CVE) of a continuous-time zero-mean Gaussian white noise signal (and any of its sub-filtered bands) is invariant and equal to $\sqrt{(4-\pi) / \pi} \approx 0.523$ (Schwartz, Bennett, and Stein, 1995). Thus, the Gaussian CVE is a universal constant and acts as a fingerprint of Gaussianity. To capture this time-variant behaviour, the signal under study is segmented and the CVE for each epoch is estimated, as well as the mean of the envelope (ME) to measure energy. As Gaussianity is a hallmark of asynchrony, any deviations from the CVE of Gaussianity reveals synchronous processes. Díaz, Razeto-Barry, et al. (2007) showed that a signal emerging from a population of phase-locked nonlinear oscillators exhibits low-CVE values, while high-CVE signals emerged only from unsteady states. Hence, CVE(morphology) and ME(energy) can be combined to produce a novel phase-space in which signals can be categorized: the envelope characterization space. This space unifies generator 
dynamics, energy and signal morphology in a single framework.

Thus, while the studies on the alpha rhythm are vast and exhaustive, all this evidence does not provide a coherent explanation of the three aforementioned characteristics stated by Adrian at the very cradle of EEG: energy, generator dynamics and waveform. To address this problem, we propose here to study the human alpha rhythm using the EEG envelope analysis.

\section{Materials and Methods}

\subsection{Data}

\subsubsection{EEG data}

We applied the envelope analysis to the EEG data of the "Leipzig Study for Mind-Body-Emotion Interactions" (LEMON) dataset (Babayan et al., 2019). This is a cross-sectional, publicly available dataset that contains resting-state EEG data from 216 subjects and is part of the MPI Leipzig Mind-Brain-Body database. The study protocol was in accordance with the declaration of Helsinki. All participants provided written consent prior to assessments and were screened for drug use, neurological, cardiovascular, and psychiatric conditions.

The recordings were obtained using 62 active electrodes, $61 \mathrm{EEG}$ channels in accordance with the 10-10 system(Oostenveld and Praamstra, 2001) and 1 VEOG electrode below the right eye. The signals were recorded with an bandpass filter between $0.015[\mathrm{~Hz}]$ and $1[\mathrm{kHz}]$ and sampled with a frequency of $2500[\mathrm{~Hz}]$. Each recording comprised 16 blocks of $60[\mathrm{~s}], 8$ blocks with eyes closed (EC) and 8 blocks with eyes open (EO). The recording began with eyes closed and blocks were interleaved. The raw data from 13 participants was discarded due to defective metadata and low data quality. Data from the remaining 203 subjects was preprocessed as follows. The data was downsampled from 2500[Hz] to $250[\mathrm{~Hz}]$ and bandpass filtered between 1-45[Hz] with a 8th order Butterworth filter. Blocks were split into EC and EO conditions for further processing. Outlier channels were rejected and time intervals with extreme deflection or frequency bursts were removed after visual inspection. Additionally, PCA and ICA was applied for further artifact rejection. The reader can consult (Babayan et al., 2019) for details. 


\subsubsection{Synthetic EEG data from a population of weakly coupled oscillators}

Synthetic EEG signals were created using the order parameter of a population of weakly coupled nonlinear oscillators following the Matthews-Mirollo-Strogatz model(P. C. Matthews, Mirollo, and Strogatz, 1991). Simulations were implemented using the DifferentialEquations.jl package (Rackauckas and Nie, 2017). A system of 800 oscillators was instantiated, whose natural frequencies followed the uniform distribution with a bandwidth $\Delta=0.8$. Solutions for the order parameter were obtained by a fourth order Runge-Kutta method(timestep $=1 / 250$ ) using three distinct coupling constant $K: 1.1$ (phase-locking), 0.1 (incoherence), and 0.9(chaos). A 24[s] epoch was isolated from each case, and white noise and pink noise were added to reproduce the normal EEG spectral background.

\subsection{3 iEEG data}

The "atlas of the normal intracranial electroencephalogram" (AN-iEEG) dataset (Frauscher et al., 2018) was also analyzed to further study the human alpha rhythm generator dynamics. The AN-iEEG dataset is a massive resting-state iEEG dataset collected from candidates for epilepsy surgery at three different hospitals in Canada and France. The recordings were obtained while the subjects were awake with their eyes closed and were selected to exclude epileptic activity. The signals were recorded using either sEEG, subdural strips, or subdural grids at the different hospitals, and the electrode locations were projected into a common brain map. Segments 60[s] long were selected visually from each recording. The data was filtered at $0.5-80[\mathrm{~Hz}]$ with a FIR filter and decimated to $200[\mathrm{~Hz}]$. An additional adaptive filter was used to reduce power-line noise. We only analyzed signals coming from the occipital lobe, and thus we included the following structures (number of individuals in parenthesis): superior and middle occipital gyri (21); inferior occipital gyrus and occipital pole (23); cuneus (19); calcarine cortex (12); lingual gyrus and occipital fusiform gyrus (29). See (Frauscher et al., 2018) for extra details. 


\subsection{EEG data analysis}

We followed the pipeline reported by Díaz, Bassi, et al. (2018). All computational procedures were implemented using the Julia programming language (Bezanson et al., 2017) (https: //julialang.org). From the preprocessed data, data from two individuals were discarded as time interval rejection produced too short, highly discontinuous recordings. From this group of 201 individuals, only the channels $\mathrm{O} 1, \mathrm{Oz}$, and $\mathrm{O} 2$ from each subject were used for further manipulation. Channel length was $474.83 \pm 9.30[\mathrm{~s}]$ for the eyes-closed condition and $469.44 \pm 13.20[s]$ for the eyes-open condition.

The data was digitally filtered in the forward and backward direction with a second order Butterworth filter to isolate the alpha band. This approach effectively obtains a zero phase response and contributes to conserve the signal and its envelope waveform. Each channel was divided into $24[\mathrm{~s}$ ] epochs (6000 points per epoch) with an overlap of $50 \%$. This amounts to 22893 epochs for the eyes-closed condition and 22634 for the eyes-open condition. The Hilbert transform was computed for each epoch and its envelope obtained using the relation $e n v=\sqrt{s[n]^{2}+H(s[n])^{2}}$. Since the envelope computation results in spurious effects at both ends, 2[s] were excised from both tails of the estimated envelope. From these 20[s] envelopes, the coefficient of variation of the envelope $C V E=\sigma_{e n v} / \mu_{e n v}$ and the mean of the envelope(ME) were estimated. CVE values were color encoded using the "buda" color scheme(Crameri, 2018) as low-CVE/purple, mid-CVE/salmon, and high-CVE/gold.

\subsubsection{Surrogate Data for Gaussianity}

$10^{6}$ instances of a 6000 points vector were simulated, sampling each point from a normal standard distribution $\mathcal{N}(0,1)$. As in the experimental data, a sampling frequency of $250[\mathrm{~Hz}]$ was assumed. These in silico generated epochs were treated as previously described for the experimental data. The same filter was applied to each epoch to obtain the alpha band. The envelope was calculated, 2[s] segments were cut from both ends, and the CVE and ME values were computed. Then, the probability density function(PDF) and the cumulative density function(CDF) was calculated for the CVE values of these alpha-filtered white Gaussian noise epochs. The 0.005 and 0.995 quantiles were estimated to obtain the lower and upper limits of 
a $99 \%$ confidence interval to assess the null hypothesis $H_{0}$ : EEG epoch is indistinguishable from white Gaussian noise.

\subsubsection{Envelope characterisation space. Scatterplots, density plots and vector field}

The envelope characterization space is a phase space that includes CVE and EEG amplitude measured for each epoch. EEG amplitude is represented by the mean of the envelope(ME) which is log-transformed and normalized using each subject, each channel statistics. This phase space is visualized by scatterplots and density plots. The density plots were built estimating 2D-histograms with a 500x500 matrix. Each row and column was filtered with a 51coefficient binomial kernel and the matrix was plotted using an alternating colour/white palette to produce a contour-like plot. In addition, as the (CVE,log(ME)[z-scored]) data for each subject, each channel recordings form an independent data cloud that contains both eyes-open and eyes-closed points, it is possible to use the centroid of eyes-open and eyes-closed points to analyze the overall behavior in the envelope characterization space for each experimental condition. Thus, the centroid of each subject, each channel (CVE,log(ME)[z-scored]) data was estimated for eyes-open and eyes-closed points to evaluate the change between experimental conditions. Then, a single vector was traced from the eyes-open centroid (white dot) to the eyes-closed centroid (arrowhead). This procedure results in a vector field inside the envelope characterization space, where each single vector was categorised as having either low-CVE (purple), mid-CVE (salmon) or high-CVE (gold) based on the CVE coordinate of the eyes-closed centroid.

\subsubsection{Time-frequency analysis}

Time-frequency analysis was applied to a complete recording that did not suffer any time interval removal(960[s]). The eyes-closed and eyes-open blocks were re-concatenated to recover the initial interleaved protocol. Then, the data was divided in $20[\mathrm{~s}]$ epochs with an overlap of $50 \%$ and treated according to Prerau et al. (2017) setting the time-bandwidth=10[s] and number of tapers $=20$, resulting in a frequency resolution of $0.5[\mathrm{~Hz}]$. Only frequencies $20 \geq f \geq 1$ were kept and the spectral power was log-transformed and colour encoded (dark blue < cyan 
$<$ yellow $<$ dark red).

\subsubsection{Fourier/envelope analysis comparison}

One raw representative epoch for each CVE class was selected and re-analyzed using both Fourier analysis and envelope analysis. The spectrum for each epoch was calculated before filtering, while after filtering the CVE and energy were obtained and visualized in the envelope characterization space. The same steps were applied to the synthetic EEG data coming from a population of weakly coupled nonlinear oscillators.

\section{3 iEEG data analysis}

The iEEG data was treated as the EEG data, with some exceptions. The CVE surrogate distribution was re-calculated setting the sampling frequency to $200[\mathrm{~Hz}]$ to match the AN-iEEG sampling frequency. Filtering, segmentation, and envelope parameters computation remained untouched. 416 epochs were analyzed. In the envelope characterization space, energy values were log-transformed but not normalized, as the AN-iEEG dataset contains eyes-closed data only. Three representative epochs recorded from the calcarine cortex were used to compare Fourier analysis with envelope analysis.

\section{Results}

\subsection{EEG envelope analysis}

\subsubsection{CVE for alpha-filtered white Gaussian noise}

The main motivation behind the EEG envelope analysis rests upon the statistical characteristic of Gaussian signals and their envelopes. Therefore, we first review these mathematical foundations. For any zero-mean continuous-time Gaussian signal(and any of its filtered sub-bands) (Fig. 1.a), the respective envelope signal follows a Rayleigh distribution(Fig. 1.b). Since the coefficient of variation for the entire family of Rayleigh distributions is invariant and equal to $\sqrt{(4-\pi) / \pi} \approx 0.523$, the coefficient of variation of the envelope (CVE) of a infinite 
zero-mean Gaussian signal always display this value that acts as a fingerprint of Gaussianity (Fig. 1.c). In other words, CVE is dimensionless, and thus the CVE is a scale-independent parameter. Nonetheless, the uniqueness of $\sqrt{(4-\pi) / \pi}$ only holds for infinite signals, and the CVE distribution for discrete-time Gaussian signals must be estimated. This distribution varies both with epoch length and bandwidth(as well as sampling frequency). We first estimated the CVE probability density functions for Gaussian signals of different lengths $(5,10,20,40$ [s]) and bandwidths $(2,4,8,16[\mathrm{~Hz}])$ sampled at $250[\mathrm{~Hz}](\mathrm{Fig} .1 . \mathrm{d})$. All distribution are bell-shaped. The variance decreases and the mode tends to 0.523 as the epoch length and the bandwidth increase. On the other hand, when these two parameters decrease the mode and median depart from the fingerprint of Gaussianity towards low-CVE values and the dispersion increases as well. An equivalent trend is obtained when varying the sampling frequency(not shown). Thus, the surrogate CVE distribution allows establishing a confidence interval for the null hypothesis $H_{0}$ : epoch indistinguishable from Gaussian noise. As a consequence, three different CVE classes are also established: low-CVE, mid-CVE, and high-CVE (Fig. 1.e). Additionally, each CVE class displays distinctive signal morphologies produced by different amplitude modulation patterns, and thus reflect distinct underlying generative processes(Fig. 1.f).

\subsubsection{Alpha-band CVE confidence interval and CVE for resting state EEG}

To characterize the human alpha rhythm using the CVE, we first obtained confidence intervals of Gaussianity to test the experimental resting-state EEG epochs. For our simulations, we used $24[\mathrm{~s}]$ long epochs with a passband in $\approx 8-13$ following the definition of Kane et al. (2017). As expected, the CVE probability density function for alpha-filtered white Gaussian noise epochs obtained in silico is bell-shaped and unimodal with mean $=0.520$, which is near the fingerprint of Gaussianity $\sqrt{(4-\pi) / \pi} \approx 0.523$ (Fig. 2, gray trace, both panels). The 0.005 and 0.995 quantiles in this distribution are 0.460 and 0.586 , respectively. These values define the 99 $\%$ confidence interval for our hypothesis of Gaussianity and establish three CVE classes: low (CVE $<0.460)$, mid $(0.460 \leq C V E \leq 0.586)$ and high $(0.586<C V E)$. Thus, any epoch with a CVE value inside the interval $[0.460,0.586]$ is considered to be indistinguishable from Gaussian noise. The CVE distributions for eyes-open and eyes-closed experimental data is shown in Fig. 1. While both distributions exhibit positive skewness and have their medians and modes 
inside the Gaussian region, they differ in their relative contribution of each CVE class. Eyesopen data (right panel, black trace) display values almost exclusively in the mid-CVE and highCVE intervals. On the other hand, eyes-closed data (left panel, black trace) display values in all three CVE intervals, with $\approx 10 \%$ of its values in the low-CVE region. .

\subsubsection{Envelope characterization space and vector field}

The envelope characterization is a two-dimensional phase space based on two envelope signal parameters: coefficient of variation of the envelope (CVE) and mean of envelope (ME). Both values have to be combined in a single framework as the CVE is a scale-independent statistic. Scatterplots in Fig. 3 show the envelope characterization space for both conditions (all channels together). In both conditions, epochs with the lowest amplitude display CVE values inside the Gaussian region. While eyes-closed data (blue) exhibit high amplitude epochs in all three CVE regions, low-CVE epochs group around one standard deviation above the mean. On the other hand, mid-CVE and high-CVE epochs are more broadly distributed over the high energy region. Eyes-open data (orange) mainly contain low amplitude epochs (as expected from the experimental condition), displaying almost exclusively mid-CVE and high-CVE values. Density plots permit further analysis of the envelope characterization space(Fig. 4). Eyesclosed data (blue) strongly cluster in the Gaussian region around 1 standard deviation above the mean. While eyes-open data (orange) also gathers in the Gaussian region, most epochs group around 1 standard deviation below the mean. This difference of about 2 standard deviations in the overall amplitude change between conditions is also found when data from each subject, each channel is analyzed independently. Vectors from the eyes-open centroid to the eyes-closed centroid were traced and plotted in the envelope characterization space(Fig. 5). Each of these vectors was classified as either low-CVE, mid-CVE or high-CVE based on the CVE coordinate of the eyes-closed centroid. While there is some variability in the magnitude and phase of these vectors(specially high-CVE vectors), the overall trend is an amplitude change of about 2 standard deviations in all three classes of vectors. The presence of lowCVE channels shows that some recordings are intrinsically rhythmic, although most channels are either Gaussian or pulsating. 


\subsubsection{Relation between experimental condition, spectrogram, and CVE}

The spectrogram of a complete recording(960[s], no interval removal) shows that alpha power increases during the eyes-closed condition regardless of the CVE values involved (Fig. 6,left panel). CVE is found to move freely across the three CVE intervals, even though the eyesclosed centroid of the recording lies in the high-CVE region. During the eyes-open condition CVE tends to settle inside the Gaussian region. Spurious high-CVE epochs are often found in the bridge between conditions as those epoch contain both eyes-open(low energy) and eyesclosed (high energy) data, thus appearing pulsating. Three representative eyes-closed epochs associated with high alpha power display the morphology/amplitude modulation characteristic of their respective CVE values (Fig. 6,right panel).

\subsubsection{Fourier/envelope analysis comparison}

As some authors have argued in favour of time domain methods over frequency domain methods to study brain rhythms (Jones, 2016), we re-analyzed three representative epochs (same data in Fig. 6) using Fourier analysis and envelope analysis(Fig. 7). A closer inspection of their pre-filtering spectra reveals that all contain the canonical spectral peak at $\approx 10[\mathrm{~Hz}]$ (Fig. 7.b). Furthermore, their spectral profiles are almost indistinguishable from each other. The CVE, however, can easily discriminate between these epochs, as it is shown in the envelope characterization space (Fig. 7.c). A similar trend was obtained analyzing synthetic EEG signals obtained from the order parameter of the MMS model. While the three alpha-filtered epochs display different morphologies (Fig. 7.d), their pre-filtering spectra cannot be separated from each other (Fig. 7.e). Noticeably, the spectral peak at $\approx 10[\mathrm{~Hz}]$ is much narrower than the experimental one. Again, these three synthetic epochs can be recognized by their CVE in the envelope characterization space (Fig. 7.f).

\section{2 iEEG envelope analysis}

Occipital data from the AN-iEEG dataset is highly clustered in the Gaussian region, while a small percentage of epochs stretches into both synchronous regions (Fig. 8, left panel). We also analyzed AN-iEEG data recorded from the calcarine cortex independently(Fig. 8, right 
panel), as Lopes Da Silva and Storm Van Leeuwen (1977) pointed to the calcarine cortex as a possible generator for the alpha rhythm. Similarly, calcarine cortex data almost exclusively contains Gaussian epochs, being the amount of synchronous epochs negligible.

The concurrent application of Fourier analysis and envelope analysis to calcarine cortex data mirrors the results found analyzing the experimental and synthetic EEG data(Fig. 9). The spectral profile is almost the same for all epochs, but the spectral peak at $\approx 10[\mathrm{~Hz}]$ is much broader than the experimental and synthetic counterparts(Fig. 9.b). Nonetheless, these three iEEG epochs display different amplitude modulation regimes; a feature that can be revealed by the CVE (Fig. 9.c).

\section{Discussion}

\subsection{Human alpha rhythm's CVE}

CVE is a hallmark of amplitude modulation patterns that reflects underlying generative processes. Thus, in this study we used CVE to study the human alpha rhythm generator dynamics - a long-standing problem that can be traced back to the very cradle of the EEG field. Indeed, while Lord Adrian claimed before the Physiological Society that the alpha rhythm originated from synchronous cortical populations, Japanese neuroscientist Dr. Yoiki Motokawa defended (in German) his theory about EEG Gaussianity. Interestingly, most EEG epochs analyzed in this work carried the hallmark of Gaussianity, thus supporting Motokawa's interpretation of asynchrony. An important amount of epochs, however, were found to be highly rhythmic, as shown by their very low-CVE values, in accordance with Lord Adrian's interpretation of neural synchronization. Nonetheless, while it is important to characterize the alpha rhythm signal at the scalp level, it is unclear how well these signals reflect the underlying neural process on the visual cortex. EEG signals are a linear superposition of several underlying processes, blurred by volume conduction, and while Lopes Da Silva and Storm Van Leeuwen (1977) reported in dogs that the purest alpha rhythm signals are recorded from the calcarine cortex, EEG signals recorded from occipital electrodes may well contain foreign components unrelated to the alpha rhythm. Remarkably, most iEEG calcarine cortex epochs were found to be Gaussian, 
suggesting that synchronous signals found at the scalp level could have been produced by volume conduction (but iEEG sample size was much smaller). Concurrent EEG-fMRI alpha rhythm studies have showed that the increase of alpha power correlates with a decrease in occipital BOLD activity (Goldman et al., 2002; Laufs et al., 2003), although Gonçalves et al. (2006) warned against ignoring the inter- and intra-subject variability in this signal. Nevertheless, the difference (if any) in BOLD signal between Gaussian and synchronous cortical regimes is yet to be delineated, and thus the relation between neural (metabolic) activity and alpha rhythm generator dynamics is an open question.

\subsection{Event-related synchronization and desynchronization}

Lord Adrian's prestige allowed the alpha rhythm to become of age under the reluctant eyes of the scientific community, setting aside any concerns triggered by Dr. Berger's education(Gloor, 1969). Hence, it is understandable that his interpretation of the alpha rhythm as a synchronous phenomenon totally permeated the discipline, thus giving birth to concepts such as event-related synchronization(ERS) and desynchronization(ERD) (Rao and Edwards, 2008; Pfurtscheller and Lopes Da Silva, 1999). Our results, however, suggest that a review of these two fundamental concepts in EEG interpretation may be needed at this point. In particular, it is customary to associate the appearance of signals such as the alpha rhythm or the mu rhythm to phase-locking regimes, assuming that synchronization only can attain the amplitudes recorded at the level of the scalp, while their disappearance is attributed to destructive interference due to desynchronization. While it has been shown that neural synchronization does increase EEG amplitude (Musall et al., 2014), to believe that asynchrony is tantamount to self-destruction is erroneous as it is almost impossible in a population of independent oscillators that phases cancel each other perfectly. In fact, amplitude death is a well-known regime that is only produced in coupled populations (P. C. Matthews, Mirollo, and Strogatz, 1991; Saxena, Prasad, and Ramaswamy, 2012). The amplitude of a signal cannot solely account for the underlying neural dynamics as the number of neural elements recruited (Elul, 1972) or the coupling constant(Breakspear, Heitmann, and Daffertshofer, 2010) can change over time. That is precisely why an scale-independent parameter such as the CVE is needed to charac- 
terize these processes. Our results suggest that it is energy rather than neural dynamics what accounts for the alpha rhythm event-related activity.

In the same line, it is also common to associate rhythmic signals with marked spectral peaks over the 1/f-like EEG background (Buzsáki, Logothetis, and Singer, 2013). While experimental low-CVE signals are in fact consistent with Wiener's interpretation of the alpha rhythm as a phase-locking signal and Kuramoto's results that a synchronous populations display a similar spectra, our simulations showed that coupled populations under other regimes also displayed the canonical spectral peak at $\approx 10[\mathrm{~Hz}]$. More importantly, the spectra of experimental EEG and iEEG signals were almost identical for rhythms, Gaussian noise, and pulsating ripples. That Fourier analysis represents signals as a linear superposition of complex sinusoids by no means supports that spectral peaks come from sinusoidal, rhythmic signals, as not only the spectrum but also the phase profile must be taken into account. The phase profile of a Gaussian signal (uniform distribution) can be easily obtained from rhythms by Fourier transform phase randomization (Theiler et al., 1992), without altering the spectral profile at all (as shown by Díaz, Bassi, et al. (2018)).

\section{Concluding remarks}

Envelope analysis offers an elegant time-domain method to assess Gaussianity that takes into account time-frequency uncertainty in time series. Previous studies regarding Gaussianity can now be reassessed by envelope analysis. Furthermore, envelope analysis puts neural synchronization and Gaussianity in the same framework, thus allowing the continuous monitoring of neural processes purely by CVE and opening a new door to explore dynamics in EEG and other neural signals (e.g. LFP, iEEG, MEG).

Here, we used envelope analysis to characterize the EEG human alpha rhythm signal. Our results regarding the alpha rhythm generation dynamics come to confirm the hypothesis of both Lord Adrian and Motokawa-san, thus honoring the contributions of these two great scholars to the EEG field and offering a new perspective for event-related synchronization/desynchronization. 


\section{Acknowledgements}

This work was supported by Fondecyt 1170027 to JM.

\section{References}

Adrian, Edgar Douglas and Brian HC Matthews (1934). "The Berger rhythm: potential changes from the occipital lobes in man". In: Brain 57.4, pp. 355-385.

Babayan, Anahit, Miray Erbey, Deniz Kumral, Janis D Reinelt, Andrea MF Reiter, Josefin Röbbig, H Lina Schaare, Marie Uhlig, Alfred Anwander, Pierre-Louis Bazin, et al. (2019). "A mind-brain-body dataset of MRI, EEG, cognition, emotion, and peripheral physiology in young and old adults". In: Scientific data 6.1, pp. 1-21.

Berger, Hans (1929). “Über das elektroenkephalogramm des menschen”. In: Archiv für psychiatrie und nervenkrankheiten 87.1, pp. 527-570.

Bezanson, Jeff, Alan Edelman, Stefan Karpinski, and Viral B Shah (2017). "Julia: A fresh approach to numerical computing". In: SIAM review 59.1, pp. 65-98.

Breakspear, Michael, Stewart Heitmann, and Andreas Daffertshofer (2010). "Generative models of cortical oscillations: neurobiological implications of the Kuramoto model". In: Frontiers in human neuroscience 4, p. 190.

Buzsáki, György, Costas A Anastassiou, and Christof Koch (2012). "The origin of extracellular fields and currents-EEG, ECoG, LFP and spikes". In: Nature reviews neuroscience 13.6, pp. 407-420.

Buzsáki, György, Nikos Logothetis, and Wolf Singer (2013). "Scaling brain size, keeping timing: evolutionary preservation of brain rhythms". In: Neuron 80.3, pp. 751-764.

Cohen, Michael X (2017). "Where does EEG come from and what does it mean?" In: Trends in neurosciences 40.4, pp. 208-218.

Cole, Scott R and Bradley Voytek (2017). "Brain oscillations and the importance of waveform shape". In: Trends in cognitive sciences 21.2, pp. 137-149.

Crameri, Fabio (2018). "Scientific colour-maps". In: Zenodo. DOI: 10.5281/zenodo. 449129. 
Davis, Hallowell and Pauline A Davis (1936). "Action potentials of the brain: In normal persons and in normal states of cerebral activity". In: Archives of Neurology \& Psychiatry 36.6, pp. 1214-1224.

Díaz, Javier, Alejandro Bassi, Alex Coolen, Ennio A Vivaldi, and Juan-Carlos Letelier (2018). "Envelope analysis links oscillatory and arrhythmic EEG activities to two types of neuronal synchronization". In: Neuroimage 172, pp. 575-585.

Díaz, Javier, Pablo Razeto-Barry, Juan-Carlos Letelier, John Caprio, and Juan Bacigalupo (2007). "Amplitude modulation patterns of local field potentials reveal asynchronous neuronal populations". In: Journal of Neuroscience 27.34, pp. 9238-9245.

Dick, Donald E and Avery O Vaughn (1970). "Mathematical description and computer detection of alpha waves". In: Mathematical Biosciences 7.1-2, pp. 81-95.

Elul, Rafael (1972). "The genesis of the EEG". In: International review of neurobiology 15, pp. 227-272.

Frauscher, Birgit, Nicolas Von Ellenrieder, Rina Zelmann, Irena Doležalová, Lorella Minotti, André Olivier, Jeffery Hall, Dominique Hoffmann, Dang Khoa Nguyen, Philippe Kahane, et al. (2018). "Atlas of the normal intracranial electroencephalogram: neurophysiological awake activity in different cortical areas". In: Brain 141.4, pp. 1130-1144.

Gloor, Pierre (1969). "Hans Berger on electroencephalography". In: American Journal of EEG Technology 9.1, pp. 1-8.

Goldman, Robin I, John M Stern, Jerome Engel Jr, and Mark S Cohen (2002). "Simultaneous EEG and fMRI of the alpha rhythm". In: Neuroreport 13.18, p. 2487.

Gonçalves, Sonia I, Jan C De Munck, Petra JW Pouwels, Ruurd Schoonhoven, Joost PA Kuijer, Natasha M Maurits, Johannes M Hoogduin, Eus JW Van Someren, Rob M Heethaar, and FH Lopes Da Silva (2006). "Correlating the alpha rhythm to BOLD using simultaneous EEG/fMRI: inter-subject variability”. In: Neuroimage 30.1, pp. 203-213.

Jones, Stephanie R (2016). 'When brain rhythms aren't 'rhythmic': implication for their mechanisms and meaning". In: Current Opinion in Neurobiology 40, pp. 72-80.

Kane, Nick, Jayant Acharya, Sandor Beniczky, Luis Caboclo, Simon Finnigan, Peter W Kaplan, Hiroshi Shibasaki, Ronit Pressler, and Michel JAM van Putten (2017). "A revised glossary of terms most commonly used by clinical electroencephalographers and updated proposal 
for the report format of the EEG findings. Revision 2017". In: Clinical neurophysiology practice 2, p. 170.

Kuramoto, Yoshiki (1975). "Self-entrainment of a population of coupled non-linear oscillators". In: International symposium on mathematical problems in theoretical physics. Springer, pp. 420-422.

Lachaux, Jean-Philippe, Eugenio Rodriguez, Jacques Martinerie, and Francisco J Varela (1999).

"Measuring phase synchrony in brain signals". In: Human brain mapping 8.4, pp. 194-208.

Laufs, Helmut, Andreas Kleinschmidt, Astrid Beyerle, Evelyn Eger, Afraim Salek-Haddadi, Christine Preibisch, and Karsten Krakow (2003). "EEG-correlated fMRI of human alpha activity". In: Neuroimage 19.4, pp. 1463-1476.

Lopes Da Silva, FH and W Storm Van Leeuwen (1977). "The cortical source of the alpha rhythm". In: Neuroscience letters 6.2-3, pp. 237-241.

Lopes Da Silva, FH, THMT Van Lierop, CF Schrijer, and W Storm Van Leeuwen (1973). "Organization of thalamic and cortical alpha rhythms: spectra and coherences". In: Electroencephalography and clinical neurophysiology 35.6, pp. 627-639.

Matthews, Paul C, Renato E Mirollo, and Steven H Strogatz (1991). "Dynamics of a large system of coupled nonlinear oscillators". In: Physica D: Nonlinear Phenomena 52.2-3, pp. 293-331.

Motokawa, Koiti (1943). "Eine statistisch= mechanische Theorie über das Elektrenke= phalogramm". In: The Tohoku Journal of Experimental Medicine 45.3-4, pp. 278-296.

Motokawa, Koiti and Tosisada Mita (1942). "Das Wahrscheinlichkeitsprinzip über die gehirn elektrische Erscheinungen des Menschen". In: Japanese journal of medical sciences III Biophysics 8, pp. 63-77.

Musall, Simon, Veronika von Pföstl, Alexander Rauch, Nikos K Logothetis, and Kevin Whittingstall (2014). "Effects of neural synchrony on surface EEG". In: Cerebral Cortex 24.4, pp. 1045-1053.

Niedermeyer, Ernst (2005). "The normal EEG of the waking adult". In: Electroencephalography: Basic principles, clinical applications, and related fields. Ed. by Niedermeyer E and Lopes da Silva FH. Lippincott Williams \& Wilkins Philadelphia, pp. 155-164. 
Oostenveld, Robert and Peter Praamstra (2001). "The five percent electrode system for highresolution EEG and ERP measurements". In: Clinical neurophysiology 112.4, pp. 713-719. Pfurtscheller, G and FH Lopes Da Silva (1999). "Event-related EEG/MEG synchronization and desynchronization: basic principles". In: Clinical neurophysiology 110.11, pp. 1842-1857.

Prast, JW (1949). "An interpretation of certain EEG patterns as transient responses of a transmission system". In: Electroencephalography and clinical neurophysiology 1.1-4, p. 370.

Prerau, Michael J, Ritchie E Brown, Matt T Bianchi, Jeffrey M Ellenbogen, and Patrick L Purdon (2017). "Sleep neurophysiological dynamics through the lens of multitaper spectral analysis". In: Physiology 32.1, pp. 60-92.

Rackauckas, Christopher and Qing Nie (2017). "DifferentialEquations.jl-a performant and featurerich ecosystem for solving differential equations in julia". In: Journal of Open Research Software 5.1.

Rao, R and E Edwards (2008). "Faculty Opinions Recommendation of [Díaz J et al., J Neurosci 2007 27(34):9238-9245]". In: Faculty Opinions. DOI: 10 .3410/f .1127024.584154.

Saunders, Michael G (1963). "Amplitude probability density studies on alpha and alpha-like patterns". In: Electroencephalography and clinical Neurophysiology 15.5, pp. 761-767.

Saxena, Garima, Awadhesh Prasad, and Ram Ramaswamy (2012). "Amplitude death: The emergence of stationarity in coupled nonlinear systems". In: Physics Reports 521.5, pp. 205228.

Schroeder, MJ and RE Barr (2000). "An alpha modulation index for electroencephalographic studies using complex demodulation". In: Medical and Biological Engineering and Computing 38.3, pp. 306-310.

Schwartz, Mischa, William R Bennett, and Seymour Stein (1995). Communication systems and techniques. John Wiley \& Sons.

Singer, Wolf (1999). "Neuronal synchrony: a versatile code for the definition of relations?" In: Neuron 24.1, pp. 49-65.

Strogatz, Steven H (1994). "Norbert Wiener's brain waves". In: Frontiers in mathematical biology. Springer, pp. 122-138. 
Theiler, James, Stephen Eubank, André Longtin, Bryan Galdrikian, and J Doyne Farmer (1992). "Testing for nonlinearity in time series: the method of surrogate data". In: Physica D: Nonlinear Phenomena 58.1-4, pp. 77-94.

Varela, Francisco, Jean-Philippe Lachaux, Eugenio Rodriguez, and Jacques Martinerie (2001). "The brainweb: phase synchronization and large-scale integration". In: Nature reviews neuroscience 2.4 , pp. 229-239.

Wiener, Norbert (1948). Cybernetics or control and communication in the animal and the machine. Technology Press. 


\section{Figures}
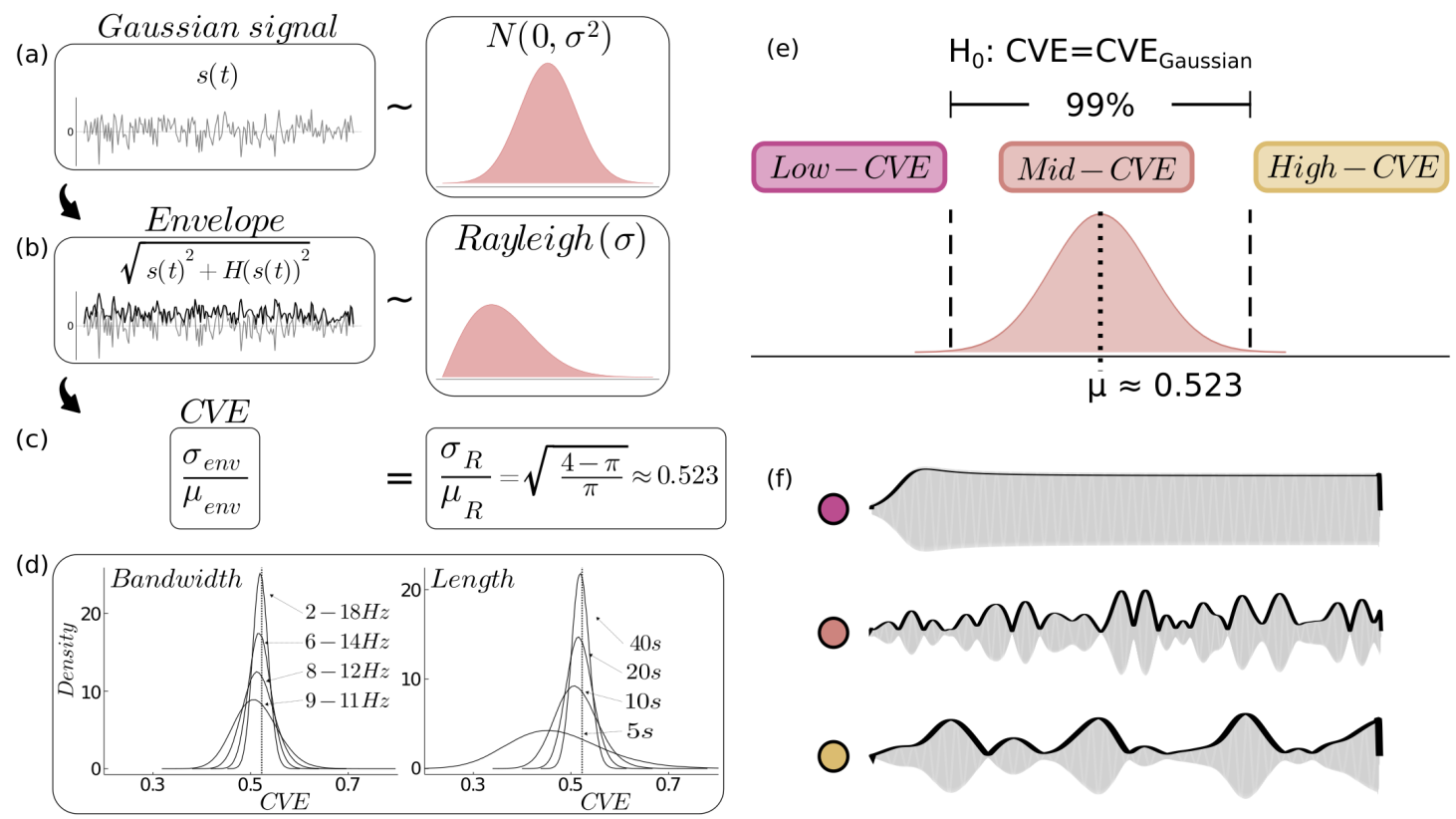

(f)

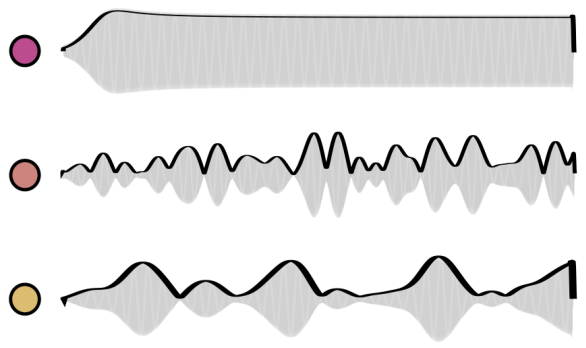

Figure 1: Coefficient of variation of the envelope is a fingerprint of Gaussianity. (a) The continuous-time Gaussian white noise signal $s(t)$ follows a Gaussian distribution $N \sim\left(0, \sigma^{2}\right)$. (b) The envelope env $(t)$ is the norm of the analytic signal $z(t)=s(t)+i H(s(t))$, where $H()$ is the Hilbert transform. Thus, env $(t)=\|z(t)\|=\sqrt{s(t)^{2}+H(s(t))^{2}}$. The envelope of $s(t)$ follows the Rayleigh distribution. (c) The coefficient of variation of the envelope (CVE) of $s(t)$ is the coefficient of variation of the Rayleigh distribution. This value is invariant and equal to $\sqrt{(4-\pi) / \pi} \approx 0.523$. (d) Since the uniqueness of $\sqrt{(4-\pi) / \pi}$ only holds for continuous-time signals, the CVE distribution for discrete-time white Gaussian noise epochs of arbitrary length is obtained in silico. CVE distributions for 24[s] epochs filtered at different bandwidths (left panel) show a mode $\rightarrow 0.523$ (dotted line) and a variance $\rightarrow 0$ as the bandwidth $\rightarrow+\infty$. CVE distributions for arbitrary length epochs filtered between 7-14 (right panel) show the same trend. The mode $\rightarrow 0.523$ and the variance $\rightarrow 0$ as the epoch length $\rightarrow+\infty$. All epochs were sampled at 250[Hz]. Notice these distributions also vary with the sampling frequency(not shown). (e) The CVE distribution for discrete-time Gaussian noise allows making a confidence interval(both dashed lines) to assess the null hypothesis of Gaussianity. Hence, three different CVE classes are established: low-CVE (purple), mid-CVE (salmon), and high-CVE (gold). (f) CVE classes correspond to distinctive amplitude modulation patterns that are linked to different generative processes. Low-CVE signals are synchronous, rhythmic signals coming from phase-locking processes, while mid-CVE signals correspond to Gaussian noise. HighCVE signals are also synchronous, albeit distinctively non-rhythmic and pulsating. 

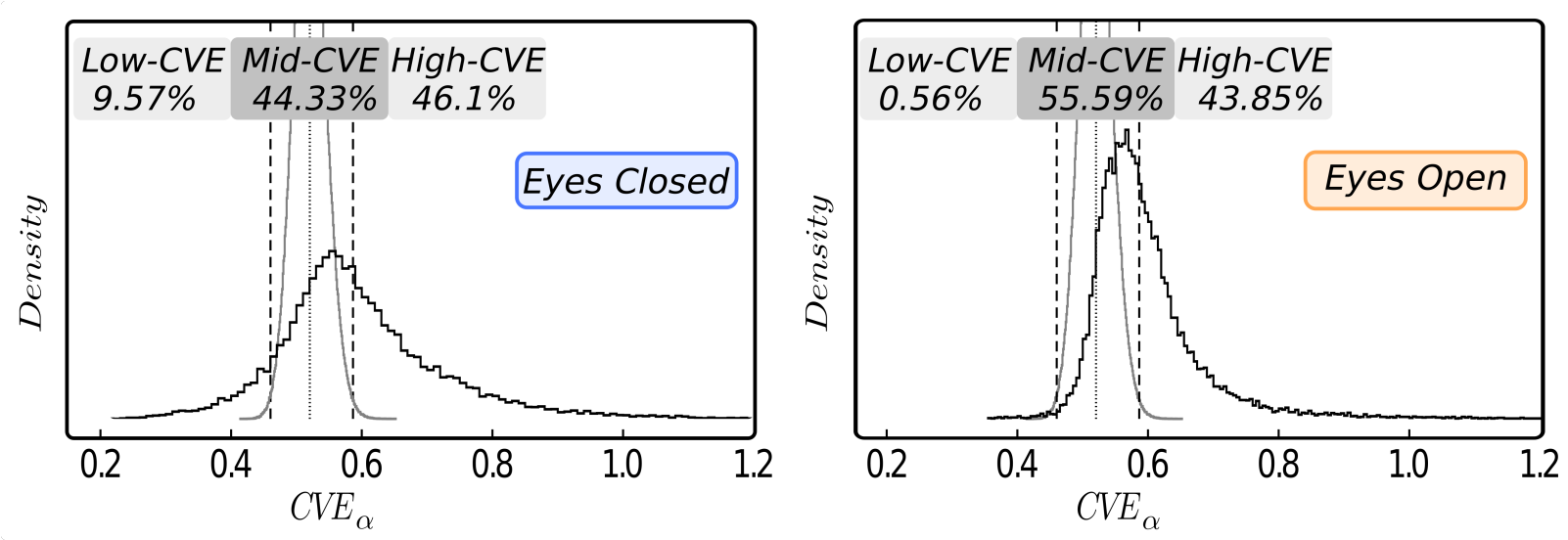

Figure 2: CVE distribution for surrogate and experimental data. The CVE distribution of alpha-filtered white Gaussian noise epochs(grey trace, both panels) shows a mean equal to 0.520(dotted line). Dashed lines at 0.460 and 0.586 depict the $99 \%$ confidence interval for Gaussianity. Both empirical distributions (black traces) are unimodal and show a mode and median inside the confidence interval for Gaussianity. The relative frequency of CVE values lying inside each CVE class is shown as percentages above each distribution. Left panel: The eyes-closed data distribution (22893 epochs) is nearly symmetrical with long tails toward low-CVE and high-CVE values. Almost $10 \%$ of experimental epochs have a low-CVE while $90 \%$ are in the Mid-High CVE ranges. Right panel: The eyes-open data distribution (22634 epochs) presents most of its values in the mid-CVE region and a long tail in the high-CVE interval. Low-CVE values are almost absent. 


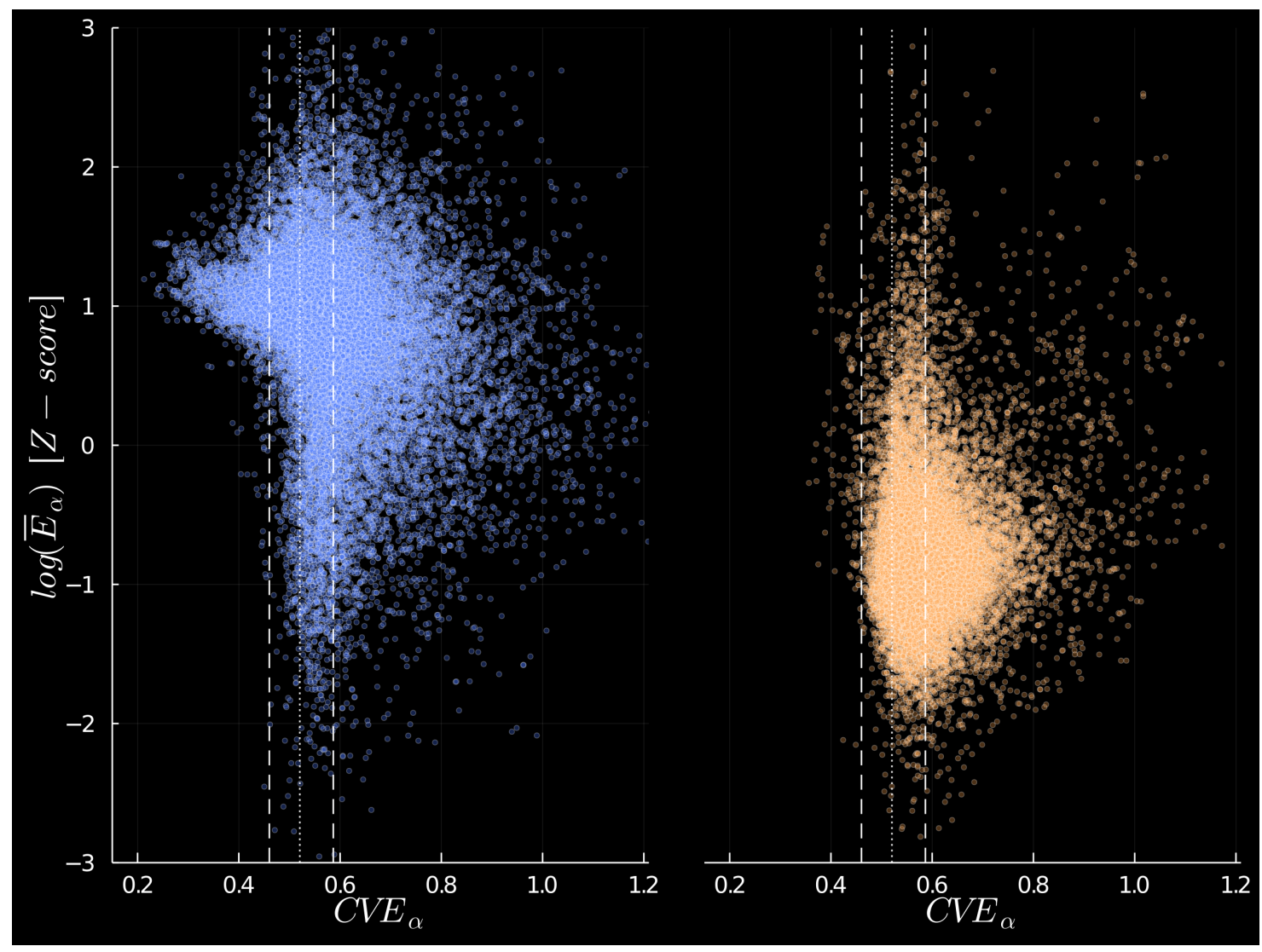

Figure 3: Scatterplot of EEG amplitude versus coefficient of variation of the envelope (CVE). The total data from 201 subjects and 3 EEG channels $(01, O z, O 2)$ was used for this plot. Each channel data was split in eyes-closed(blue) and eyes-open data(orange), filtered in the alpha band and epoched in 24[s] segments with an overlap of 12[s]. The mean of the envelope(ME) and CVE were calculated. Then, the ME was log-transformed and Z-scored using the mean and standard deviation from the whole recording(eyes-closed and eyes-open), resulting in normalized envelope amplitude values for each subject, each channel data. Left panel: Eyes-closed data (22893 epochs) show a few amplitude values below the mean energy, which corresponds to subjects whose alpha rhythm is either minimum or non-existent(as widely reported in the literature). Additionally, high energy epochs are present in all three CVE Classes. Right panel: Eyes-open data(22634 epochs) mostly show amplitude below the mean energy, as expected from the experimental condition. While these data show a clear tendency towards mid-CVE and high-CVE values, low-CVE values are almost absent. Dotted line at 0.520, the mean of the surrogate alpha-filtered white Gaussian noise CVE distribution. Dashed lines at 0.460 and 0.586 depict the $99 \%$ confidence interval for Gaussianity. Alpha channel was set to $50 \%$ to highlight cluster density. 

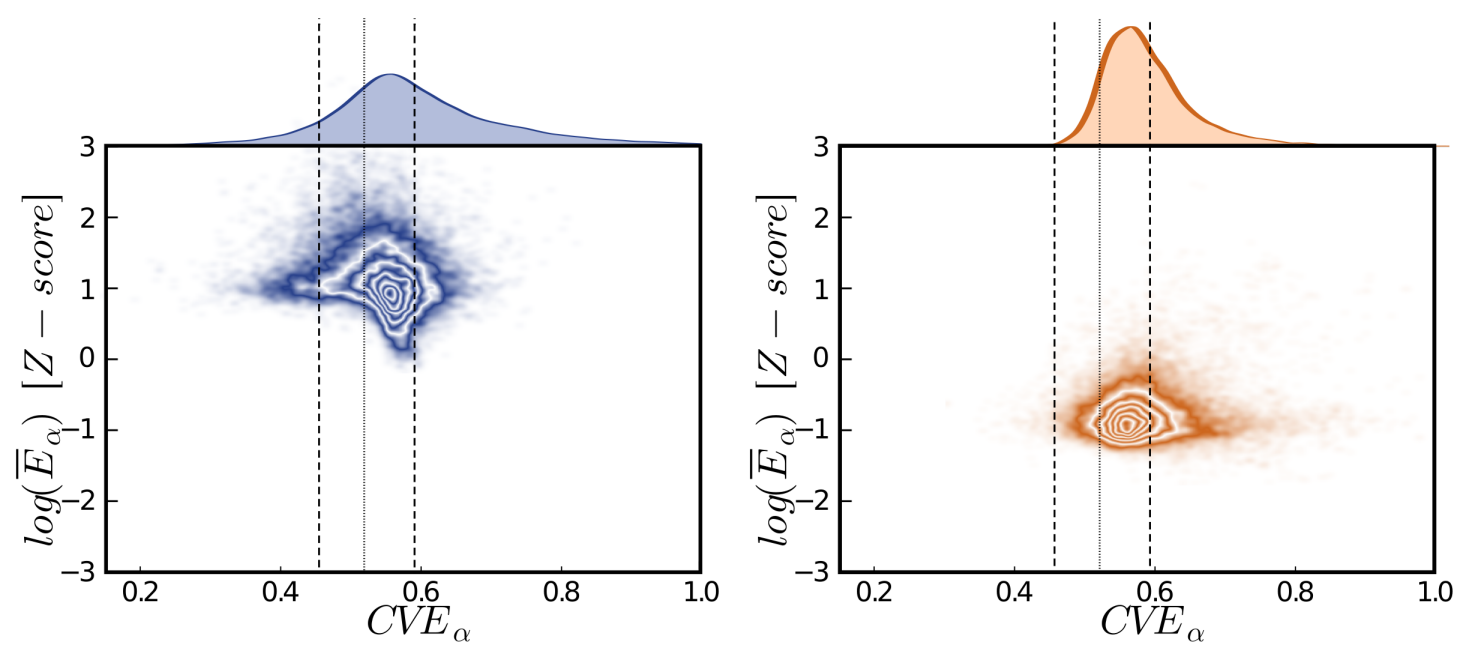

Figure 4: Empirical density functions for EEG amplitude vs CVE. Both eyes-closed(blue) and eyes-open(orange) data present just one cluster centered at similar mid-CVE values but differ in amplitude. Left panel: Eyes-closed data presents one cluster in the interval of Gaussianity with an amplitude approximately 1 standard deviation above the mean. While the great majority of epochs lie in the Gaussian region, the data display CVE values in all three intervals. Right panel: Eyes-open data also show just one cluster inside the region of Gaussianity. This cluster, however, resides in the low energy region, approximately 2 standard deviations away from the eyes-closed cluster centre. Dotted line at 0.520, the mean of the surrogate alphafiltered white Gaussian noise CVE distribution. Dashed lines at 0.460 and 0.586 depict the $99 \%$ confidence interval for Gaussianity. Maps are coloured using an alternating colour-white palette to obtain a contour-like plot. 


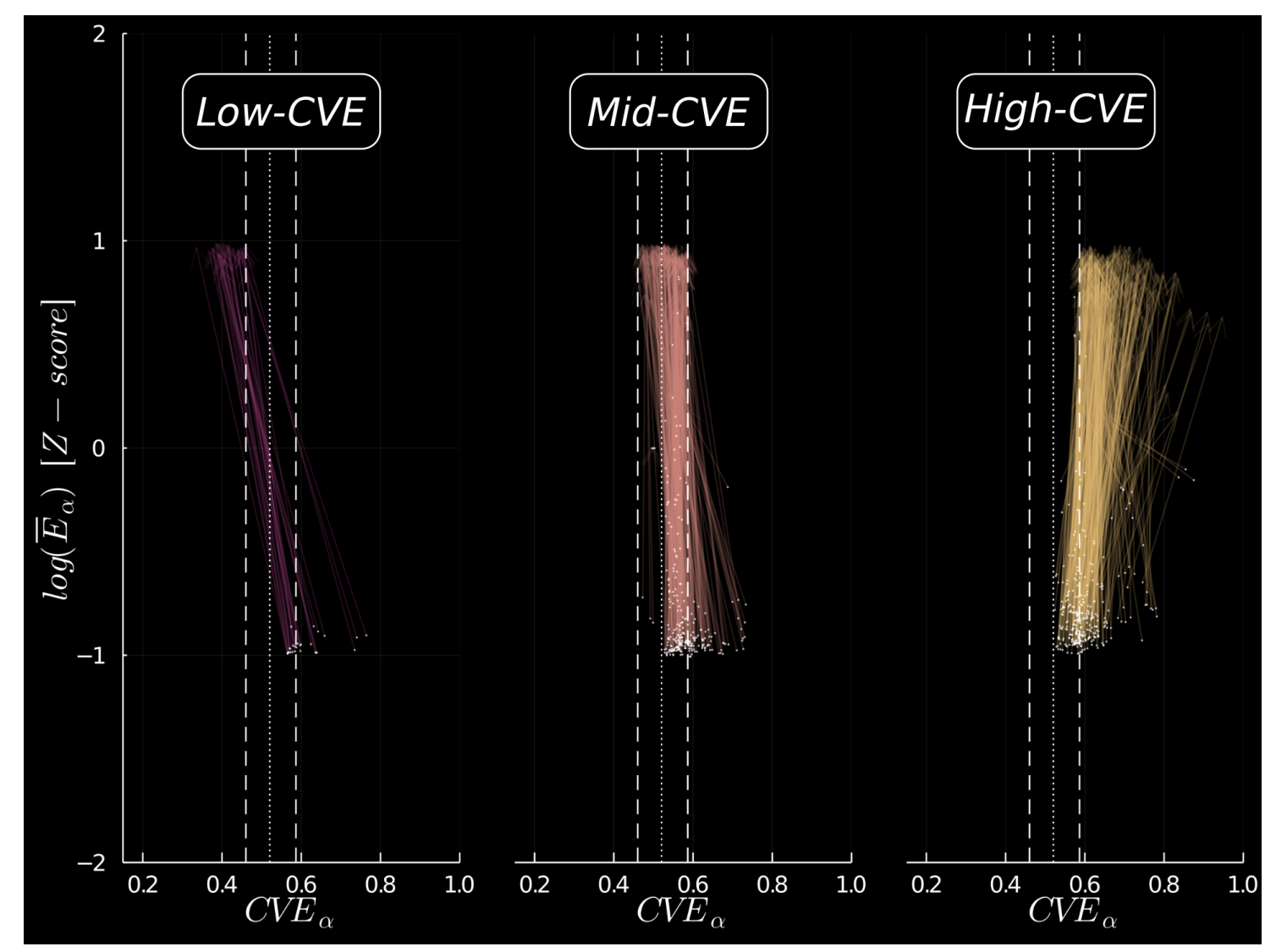

Figure 5: Envelope characterisation space vector field. The centroid of CVE and energy values from each subject $(\mathrm{N}=201)$,each channel(O1,Oz,O2) data were calculated for eyes-closed and eyes-open epochs. A single vector was calculated from the eyes-open centroid(white dot) to the eyes-closed centroid(arrowhead). Vectors were classified as either low-CVE, mid-CVE or high-CVE according to the CVE coordinate of their eyes-closed centroid, reflecting the overall behaviour of a particular subject-channel data. While almost all vectors start at mid-CVE and high-CVE centroids in the low energy range, their eyes-closed coordinates reside in all three CVE intervals with high energy. The relative frequency of vectors roughly reflects the entire population CVE distribution. Furthermore, for all three classes of vectors, the overall trend is to have an amplitude component whose absolute value $\approx 2$. Dotted line at 0.520 , the mean of the surrogate alpha-filtered white Gaussian noise CVE distribution. Dashed lines at 0.460 and 0.586 depict the $99 \%$ confidence interval for Gaussianity. Alpha channel was set to $50 \%$ to highlight cluster density. 

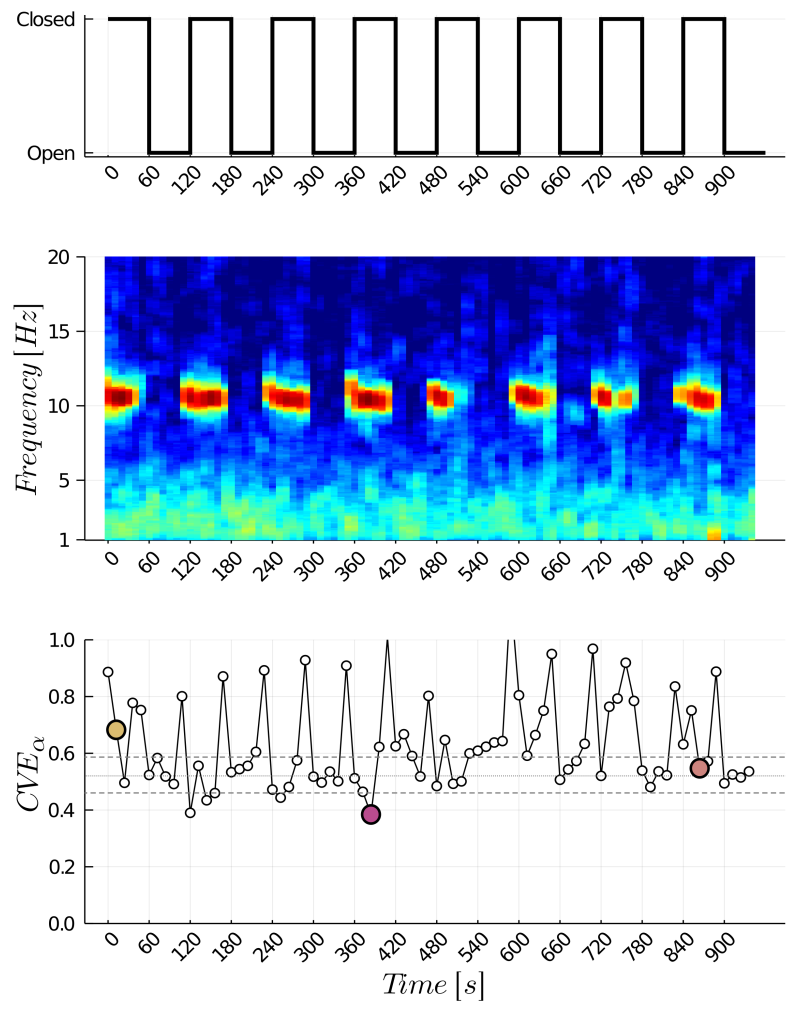
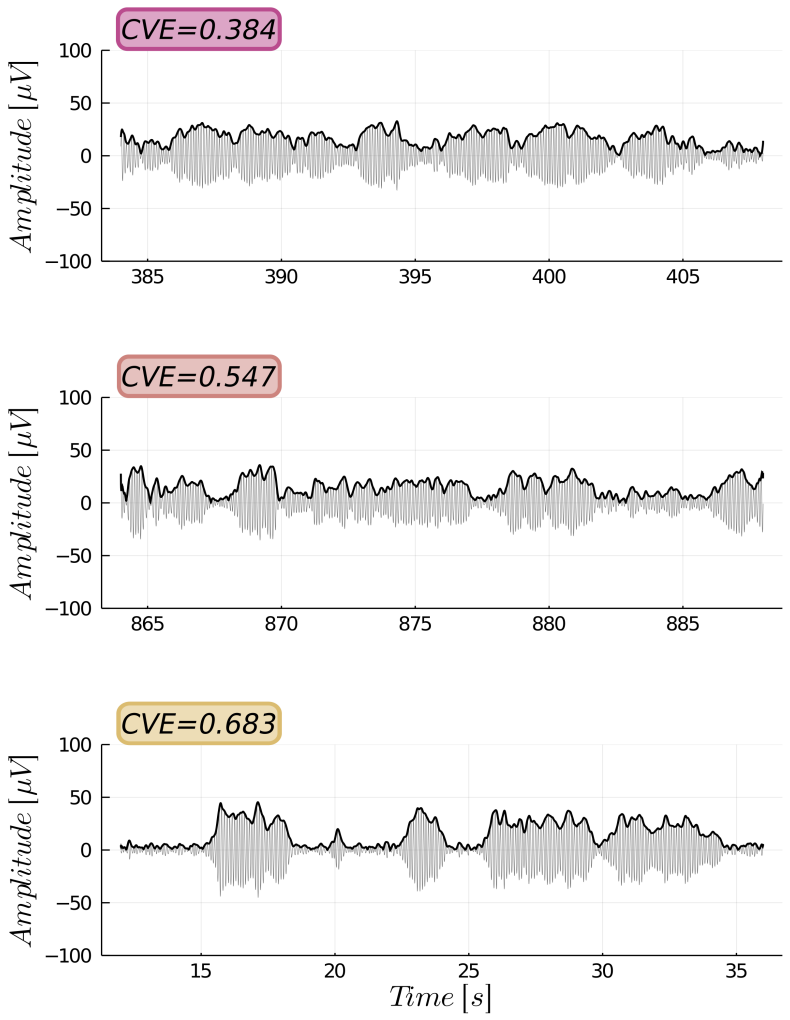

Figure 6: Relation between experimental condition, time-frequency analysis and CVE. Data correspond to a complete 960[s] recording(channel 01) from a representative individual whose eyes-closed centroid is in the high-CVE interval. Left panel: from top to bottom, experimental condition, multitaper spectrogram(data not filtered) and CVEgram(alpha-filtered data). While both conditions show a broadband 1/f-like spectrum, only the eyes-closed condition contain a narrowband component $\approx 10[\mathrm{~Hz}]$. Furthermore, the CVEgram shows low, mid and high CVE values associated with the narrowband spectrum during the eyes-closed condition. On the other hand, only mid-CVE and high-CVE values are present during the eyes-open condition. The right panel depicts three representative eyes-closed 24[s] epochs with their respective envelopes corresponding to rhythmic(upper trace), Gaussian(middle trace) and arrhythmic(lower trace) activity. Dotted line at 0.520, the mean of the surrogate alpha-filtered white Gaussian noise CVE distribution. Dashed lines at 0.460 and 0.586 depict the $99 \%$ confidence interval for Gaussianity. 

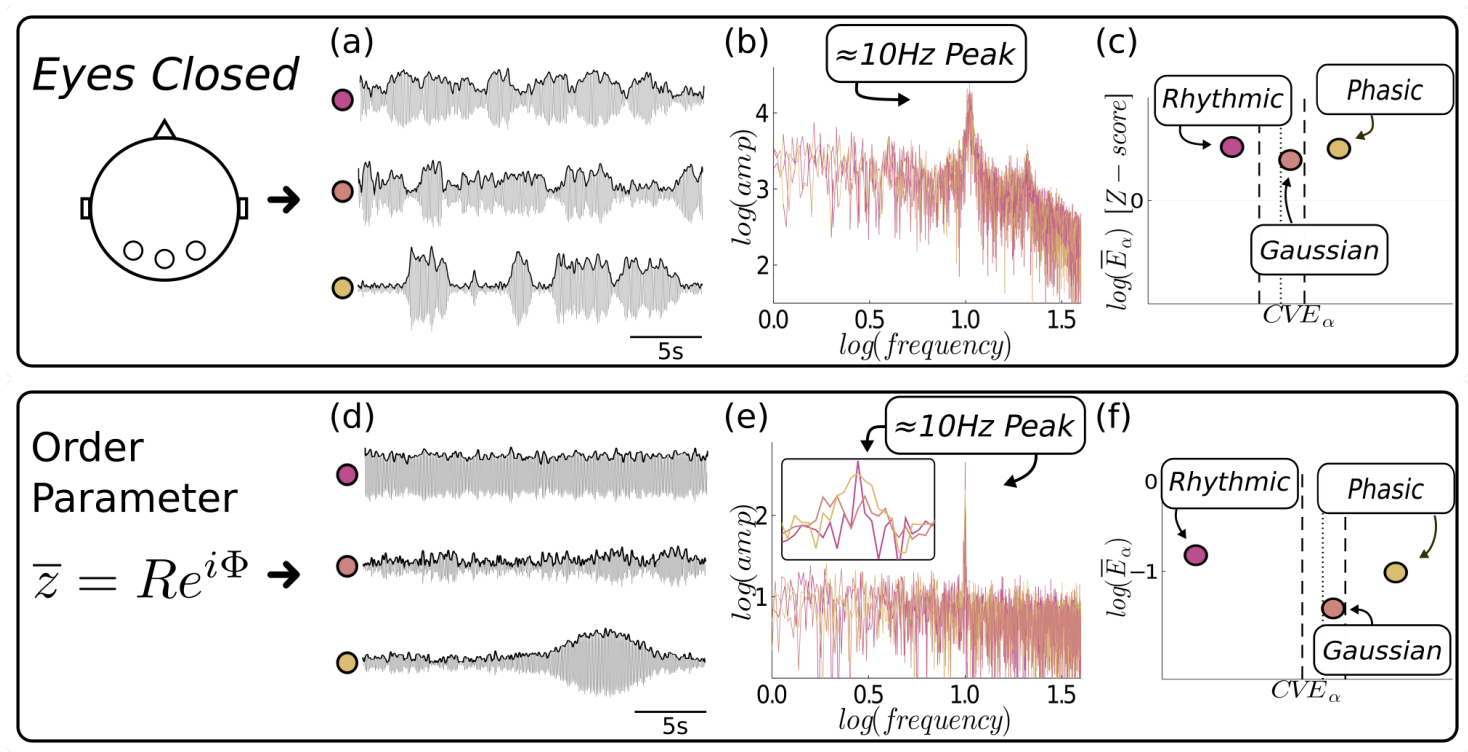

Figure 7: Envelope analysis reveals both Gaussian and synchronous dynamics in eventrelated activity. Three eyes-closed EEG epochs were re-analysed using Fourier analysis(raw data) and envelope analysis(alpha-filtered data). (a) Three experimental alpha-filtered epochs and their respective envelopes are shown(same data in Fig. 6), each epoch corresponding to one CVE class (rhythmic, Gaussian, phasic). (b) While the three epochs contain energy $\approx 10[\mathrm{~Hz}]$, their spectra are almost indistinguishable from each other. (c) On the other hand, the envelope analysis for the alpha-filtered data does discriminate between the three epochs by CVE. Dotted line at 0.520 , the mean of the surrogate alpha-filtered white Gaussian noise CVE distribution. Dashed lines at 0.460 and 0.586 depict the $99 \%$ confidence interval for Gaussianity. 


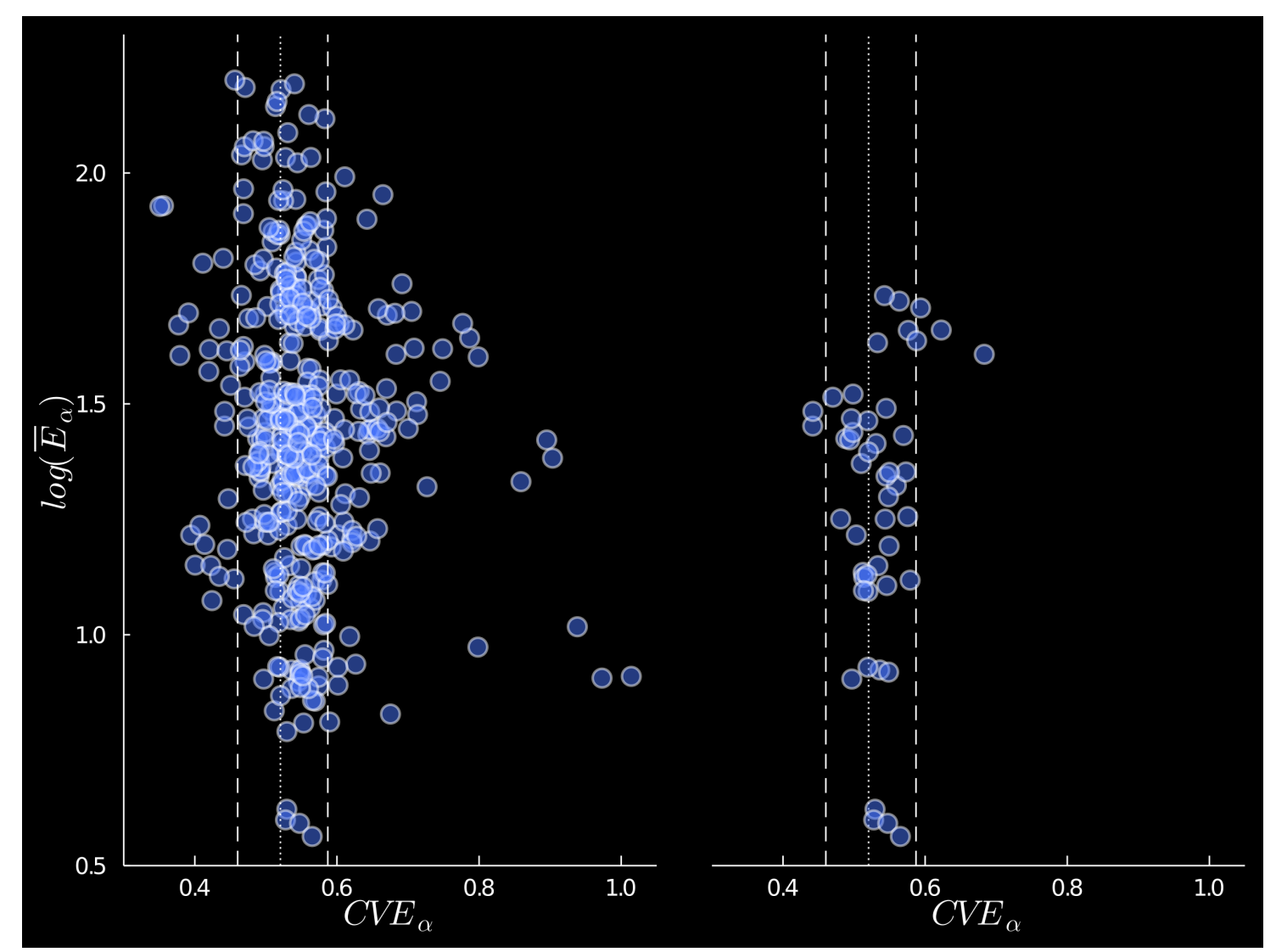

Figure 8: Scatterplot of iEEG amplitude versus coefficient of variation of the envelope (CVE). Each eyes-closed recording was filtered in the alpha band and epoched in 24[s] segments with an overlap of 12[s]. The mean of the envelope(ME) and CVE were calculated. Then, the ME was log-transformed but not normalized. Left panel: Occipital data (412 epochs) is highly clustered in the the Gaussian region, while some epochs lie inside both synchronous regions. Right panel: Calcarine cortex data (48 epochs) mostly contains Gaussian epochs as well. Synchronous epochs are practically nonexistent. Dotted line at 0.520 , the mean of the alpha-filtered white Gaussian noise CVE distribution obtained in silico. Dashed lines at 0.455 and 0.592 depict the $99 \%$ confidence interval for $H_{0}$ : epoch indistinguishable from white Gaussian noise. 


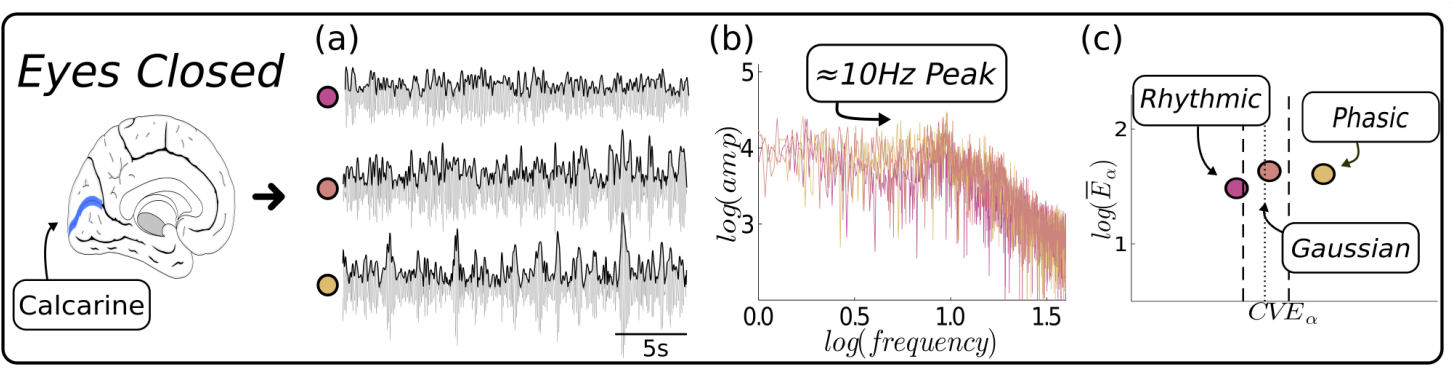

Figure 9: Envelope analysis reveals both Gaussian and synchronous dynamics in intracranial event-related activity. Three eyes-closed iEEG epochs recorded from the calcarine cortex were re-analysed using Fourier analysis(raw data) and envelope analysis(alpha-filtered data). (a) Three experimental alpha-filtered epochs and their respective envelopes are shown, each epoch corresponding to one CVE class (rhythmic, Gaussian, phasic). (b) The three epochs contain energy at $\approx 10[\mathrm{~Hz}]$ and their spectra are very similar. The spectral peak is much broader than the observed in the EEG(Fig. 7) or synthetic(Fig. 8) data. (c) The envelope analysis for the alpha-filtered data discriminates between the three epochs by CVE. Dotted line at 0.520, the mean of the surrogate alpha-filtered white Gaussian noise CVE distribution. Dashed lines at 0.460 and 0.586 depict the $99 \%$ confidence interval for Gaussianity. 